\title{
Valle Giumentina (Abruzzes)
}

Sixième mission : étude des comportements techno-économiques au Pléistocène moyen

Élisa Nicoud, Valentina Villa, Gourguène Davtian et Juliette GuibertCardin

\section{(2) OpenEdition}

\section{Journals}

Édition électronique

URL : http://journals.openedition.org/cefr/1891

DOI : 10.4000/cefr.1891

ISSN : 2282-5703

Éditeur

École française de Rome

Référence électronique

Élisa Nicoud, Valentina Villa, Gourguène Davtian et Juliette Guibert-Cardin, « Valle Giumentina (Abruzzes) », Chronique des activités archéologiques de l'École française de Rome [En ligne], Italie centrale, mis en ligne le 07 mars 2018, consulté le 01 mai 2019. URL : http://journals.openedition.org/ cefr/1891; DOI : 10.4000/cefr.1891

Ce document a été généré automatiquement le 1 mai 2019.

(c) École française de Rome 


\title{
Valle Giumentina (Abruzzes)
}

Sixième mission : étude des comportements techno-économiques au Pléistocène moyen

\author{
Élisa Nicoud, Valentina Villa, Gourguène Davtian et Juliette Guibert- \\ Cardin
}

\section{NOTE DE L'AUTEUR}

Les recherches à Valle Giumentina sont financées par l'École française de Rome, la Fondation Pescarabruzzo, le Parco nazionale della Majella, et bénéficie du soutien logistique de la commune d'Abbateggio, de l'Archéoclub de Pescara, du Museo delle Genti d'Abruzzo et de la commune de Caramanico Terme.

Les chercheurs impliqués dans le projet couvrent de nombreuses disciplines de l'étude du Quaternaire. La fouille est dirigée par Élisa Nicoud. L'étude technologique des industries lithiques est dirigée par É. Nicoud, Daniele Aureli et Marina Pagli. Membres de l'équipe de recherche : É. Nicoud, Valentina Villa, D. Aureli, M. Pagli, Giovanni Boschian, Silvano Agostini, Christine Chaussé, Paul Mazza, Maria Adelaide Rossi, Fabio Fusco, Catherine Kuzucuoglu, Biagio Giaccio, Marion Hernandez, Christelle Lahaye, Norbert Mercier, Cristina Lemorini, Andrea Zupancich, Antonin Tomasso, Nicole Limondin-Lozouet, Emmanuelle Stoetzel, Sébastien Nomade, Alison Pereira, JeanJacques Bahain, Amina Vietti, Eleonora Regattieri, Vincent Robert, Clément Virmoux, Juliette Guibert-Cardin, Gourgen Davtian avec la collaboration de Tommaso Colonna et le CGT Group, Andrea D’Andrea, Rosario Valentini, Marianna Fusco, Arnaud Mazuy, Alain Carré, Sabin Sorin-Mazouni, Cesare Fanelli.

En 2017, les 25 bénévoles étaient français et italiens, issus de nombreuses institutions : Guido Palmerini (Università di Chieti-Pescara), Caterina Aureli, Elena Carletti, Marianna Fusco (La Sapienza, Università di Roma), Giorgia Sardelli, Eva Martellotta (Università degli Studi di Ferrara/Erasmus CEPAM), Errico Pontis (Università degli studi di Napoli «L'Orientale»), Félicien Capellari (université de Perpignan), Morgane BardeyVaillant (Muséum national d'histoire naturelle), Élodie Reboul (université de Bordeaux), Quentin Wackenheim, Julia Bude, Alexis Noqué (université de Paris 1 Panthéon- 
Sorbonne), Julie Perrotte (université de Rennes), Ombline Grosbois (université de Nantes), Sébastien Plutniak (École française de Rome), Charlotte Pruvost, Carlo Mologni (université Côte d'Azur), Laura Haby, Pierre Gaignard, Romain Talon, Clélia Renault, Guillaume Thomas, Florian De Gasperi (Projet Idex, université Côte d'Azur).

\section{Les objectifs de la mission de terrain 2017}

1 Valle Giumentina est un gisement des Abruzzes du Paléolithique ancien et moyen en plein air, connu grâce aux travaux approfondis des années 1950 menés par le préhistorien A.M. Radmilli et le géologue J. Demangeot ${ }^{1}$. Sept horizons archéologiques avaient été mis au jour en contexte globalement lacustre, à la faveur d'une ravine profonde qui a incisé les dépôts du pléistocène jusqu'à $25 \mathrm{~m}$ de profondeur. Les recherches pluridisciplinaires entreprises dans le cadre du programme quinquennal de l'École française de Rome (2012-2016) en collaboration avec la Surintendance des Abruzzes ont permis de préciser la géochronologie du gisement, étape importante et nécessaire pour discourir sereinement des problématiques paléo-historiques, notamment au travers de l'étude des industries lithiques. Nos recherches ont réuni plus de 30 quaternaristes et nécessité en premier lieu la mise au jour d'une coupe stratigraphique (VV1) sur $17 \mathrm{~m}$ de puissance, la réalisation d'un carottage mécanique ayant atteint $45 \mathrm{~m}$ de profondeur (VG1), de profils géophysiques et d'une fouille planimétrique sur une aire de $50 \mathrm{~m}^{2}$. En 2016, les niveaux archéologiques, ALB-42, LDP-41 et LABM-40 ont déjà fait l'objet de fouille manuelle en extension sur une aire allant de 50 à $35 \mathrm{~m}^{2}$, cette diminution étant induite par la mise en place de paliers de sécurité 2 .

2 Treize niveaux archéologiques sont désormais attestés le long de la coupe VV1 (fig. 1). Ils sont compris dans une séquence stratigraphique puissante de $25 \mathrm{~m}$, ayant enregistré deux cycles glaciaires-interglaciaires, bien décrits par les études sédimentologiques et paléoenvironnementales. Les datations par méthode ${ }^{40} \mathrm{Ar} /{ }^{39} \mathrm{Ar}$ sur dépôts volcaniques lui attribuent un âge allant de 620000 ans à la base à 400000 ans pour le dernier niveau d'occupation, lui-même scellé par des dépôts glaciaires et pédogénétiques plus récents ${ }^{3}$. En octobre 2017, deux thèses de doctorat réalisées en partenariat avec l'EFR ont été soutenues, la première par Valentina Villa sur le contexte chronostratigraphique, géomorphologique et environnemental de Valle Giumentina et la seconde par Alison Pereira, sur les datations par la méthode ${ }^{40} \mathrm{Ar} /{ }^{39} \mathrm{Ar}$ des sites du Pléistocène moyen italien en contexte volcanique, en premier lieu, Valle Giumentina ${ }^{4}$. 
Fig. 1 - Coupe stratigraphique VV1 de Valle Giumentina : a. localisation générale du site ; $b$. prise de vue aérienne en drone et indications de l'emplacement des fenêtres stratigraphiques " VV » le long de la ravine ; c. coupe VV1 et datations par méthodes ${ }^{40} \mathrm{Ar} /{ }^{39} \mathrm{Ar}$ des couches encadrant le niveau SLM-37 (Pereira 2017); d. stratigraphie schématique, la flèche blanche indique le niveau SLM-37 fouillé en 2017.

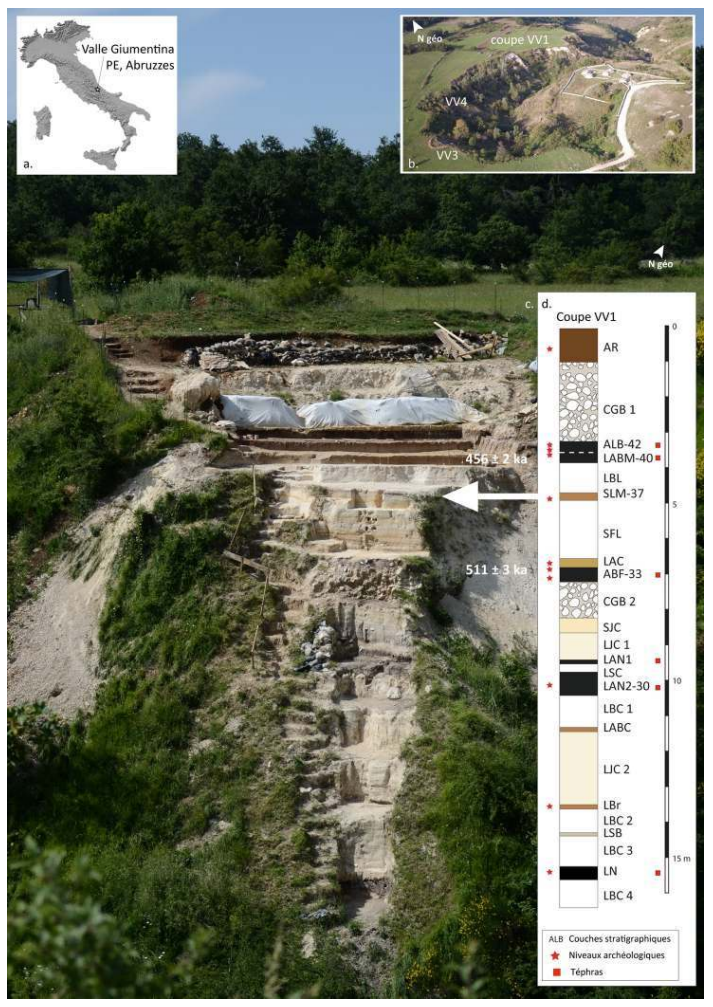

b. E. Nicoud ; c. EFR/P. Gaignard; d. Villa 2017.

3 Ainsi, la poursuite des recherches de terrain à Valle Giumentina dans le cadre du nouveau projet quinquennal 2018-2021 de l'École française peut désormais se concentrer sur les données archéologiques. La campagne de fouille 2017 (29 mai-14 juillet) a porté sur le niveau SLM-37 connu depuis les fouilles Radmilli pour sa composante d'industrie bifaciale. C'est lui qui avait déterminé l'attribution du site à la culture de l'Acheuléen, quand les autres niveaux l'encadrant, dépourvus de bifaces, étaient par conséquent attribués au Clactonien. Dans les années 1950, seul ce niveau avait livré des bifaces. Or, aujourd'hui, nous savons qu'il y a également des industries bifaciales en LABM-40 et dans les niveaux sous-jacents, SLM-37 et LAC-34. Les $35 \mathrm{~m}^{2}$ fouillés sur la coupe principale VV1 ont livré 253 industries lithiques (hors refus de tamis) dont un biface et quelques restes osseux. Tous les sédiments ont été tamisés par quart de $\mathrm{m}^{2}$ (452 unités de refus de tamis).

Le paléosol SLM (sable limoneux à mollusques) repéré dans les locus VV1 et en VV3 est bien corrélé au niveau 37 des fouilles anciennes notamment grâce à sa malacofaune extrêmement riche, caractéristique d'un milieu ouvert et aride ${ }^{5}$. Il est compris dans une épaisse succession de couches fluvio-lacustres. La pédogenèse a affecté cette couche, légèrement brunifiée, de façon moins intense que les couches ALB-42 ou LABM-40 par exemple. Les traits de bioturbation et de pédogenèse discrets qui caractérisent la couche SLM permettent de la définir comme un sol peu mature, contemporain d'une phase de relative stabilité environnementale. Sa formation peut correspondre soit à une amélioration climatique brève, soit à des conditions interstadiaires ${ }^{6}$. Les vestiges 
archéologiques et les restes de faune découverts lors des fouilles 2015 et 2016 dans le locus VV3 à la surface du sol SLM sont disposés à plat sur un niveau unique et ne présentent aucune trace d'altération. Cela indique leur position in situ et leur recouvrement rapide par les sédiments fluvio-lacustres (couche LBL sus-jacente) peu après leur dépôt.

Par ailleurs, une équipe de cinq personnes a filmé la mission dans le cadre du projet « in minimis maxima» de l'université Côte d'Azur (IDEX Jedi). Il s'agit de réaliser un film documentaire hybride voulant mêler sciences et art. Ce projet devrait se conclure en 2018.

\section{Contexte stratigraphique et paléoenvironnemental du niveau SLM-37 (V. Villa)}

6 Le niveau archéologique SLM-37 est inclus dans un des paléosols qui rythment la partie supérieure de la séquence de Valle Giumentina (ensemble stratigraphique EN1). Ce niveau se situe entre les unités stratigraphiques LBL-39/38 et SFL-36/35 (fig. 1).

7 La couche SLM, de couleur brun clair (10YR 7/3) présente une texture limono-argileuse (argiles 25-30\% ; limons 64-56 \%; sables 11-12\%) et une structure massive tassée. Les pores d'origine biologique sont assez fréquents, de dimensions réduites (inférieures au millimètre) et leurs bords sont caractérisés par des figures d'oxydations faiblement exprimées, telles des mouchetures couleur rouille. La limite inférieure est irrégulière, ondulée ${ }^{7}$.

8 La couche a été identifiée à plusieurs endroits le long de la ravine qui entaille le bassin de Valle Giumentina. Les corrélations stratigraphiques établies entre les coupes VV1, VV3 et VV4 sur la base des caractéristiques lithologiques de ces dépôts ont été vérifiées et confirmées par les analyses malacologiques réalisées sur les restes de mollusques terrestres qu'y sont conservés. Une publication dédiée aux données malacologiques est disponible ${ }^{8}$. Ces corrélations permettent de préciser la géométrie des dépôts et mettent en évidence un pendage est-ouest, également observable dans le profil stratigraphique large de $6 \mathrm{~m}$ dégagé dans l'aire de fouille archéologique.

9 En 2017, un relevé au GPS différentiel de la zone d'intervention par G. Davtian a notamment permis d'apporter des précisions quant à la localisation relative des deux aires de fouilles, VV1 et VV3 et ainsi, des différentes profondeurs d'apparition des vestiges dans ces deux secteurs. Ce relevé confirme le pendage de la couche SLM vers l'ouest (fig. 2), inverse par rapport aux pendages des couches supérieures (ALB-42 et LABM-40). Des modèles numériques de terrain ont été produits ainsi que des vues des secteurs de fouille en photogrammétrie. Enfin, des points de contact stratigraphiques à la base du remplissage lacustre ont également été relevés. Toutes ces données valables pour l'ensemble des niveaux archéologiques de Valle Giumentina sont en cours de traitement. 
Fig. 2 - Valle Giumentina niveau SLM-37 en VV1 : a. photographie de la coupe est-ouest ; b. dessins des coupes sud-nord et est-ouest (selon le nord de référence de la fouille) ; c. relevé topographique de la plateforme de fouille et localisation des coupes stratigraphiques.

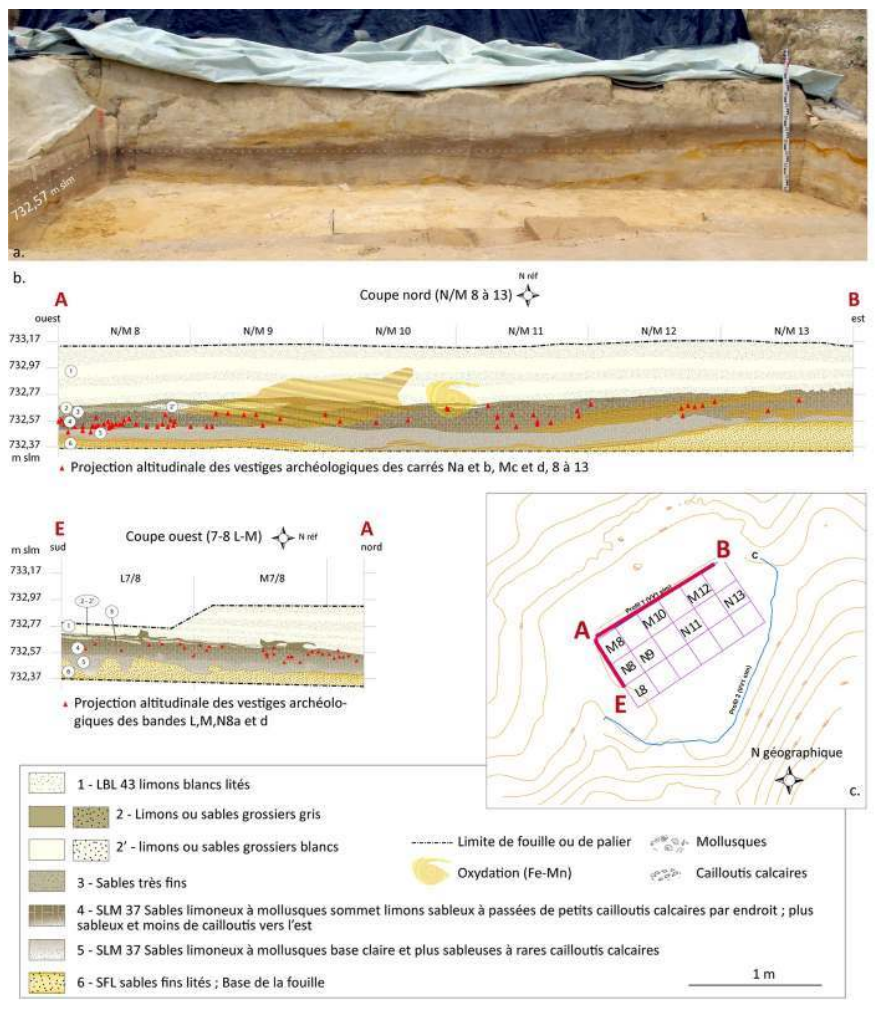

a. et b. EFR/E. Nicoud ; c. G. Davtian.

L'étude micromorphologique a été réalisée sur les échantillons prélevés en parallèle dans l'unité dans les coupes VV1 et VV3. En lame mince, la couche SLM apparaît constituée de grains détritiques calcaires qui baignent dans une matrice micritique. Par rapport à l'unité SFL sous-jacente, la fraction fine est ici beaucoup plus importante et on observe une augmentation nette des fractions argileuse et limoneuse. Quelques éléments centimétriques subarrondis de silex et de calcaire sont présents, emballés dans la matrice (fig. 3a-b). Des nodules argileux également d'origine détritique sont incorporés au sédiment. Des traits de bioturbation diffuse sont aussi détectés : une porosité constituée de chenaux et de fréquents remplissages discontinus d'excréments arrondis.

La caractéristique qui distingue cette couche est la présence de très nombreux restes de mollusques (fig. 3c). En lame mince, on observe en effet quelques coquilles entières dont les cavités sont en partie remplies de micrite (fig. 3d), de matière organique amorphe et d'oxydes ferro-manganiques (fig. 3e-f). Les figures d'oxydation restent néanmoins rares ; seules quelques intercalations d'oxydes amorphes de fer (fig. 3g-h) ont été identifiées à l'intérieur des biopores.

12 La présence d'un fragment d'os de petites dimensions ainsi que de quelques éclats de silex reconnus dans les lames minces est sans doute liée à l'occupation humaine. L'étude micromorphologique confirme donc les observations macroscopiques et les analyses sédimentologiques et permet de définir la couche SLM comme un horizon pédologique peu évolué. Les caractéristiques des couches qui encadrent ce paléosol (couches SFL et LBL) permettent d'affirmer qu'il atteste d'une phase de stabilité et d'amélioration 
climatique au cours d'une période de climat froid et aride, de type glaciaire. L'analyse malacologique conforte cette hypothèse. L'ensemble malacologique conservé dans SLM est très riche et est constitué presque exclusivement d'espèces xérophiles, telles que Truncatellina cylindrica, Cernuella cf. cisalpina et Vallonia costata, qui indiquent un milieu de type steppique, ouvert et très aride, typique d'une période glaciaire (échantillon M13 ; 3800 restes) $)^{9}$.

Cette phase glaciaire, à laquelle est attribuée une grande partie du sommet de la séquence de Valle Giumentina (ensemble EN1, couches CGB2 à CGB1), est corrélée aux MIS 12 grâce aux datations ${ }^{40} \mathrm{Ar} /{ }^{39} \mathrm{Ar}$. Il est possible d'attribuer à l'unité SLM-37 un âge compris entre $511 \pm 3 \mathrm{ka}$ et $456 \pm 2 \mathrm{ka}^{10}$. Les différents indices sédimentaires et de la malacofaune le rapportent au MIS 12c, au début du refroidissement de cette longue période glaciaire, avant le maximum glaciaire du MIS $12 a^{11}$.

Fig. 3 : Valle Giumentina 2017, paléosol SLM (coupes VV1 et VV3) : a. fragment de silex, en PPL ; $b$. la même photo que $c$ en XPL; $c$. et $d$. coquilles de mollusques, en PPL; e. coquille entière remplie de micrite et d'oxydes ferro-manganiques en PPL ; f. la même photo que e en XPL ; g. imprégnation d'oxydes ferro-manganiques en PPL; $h$. détail de $g$.
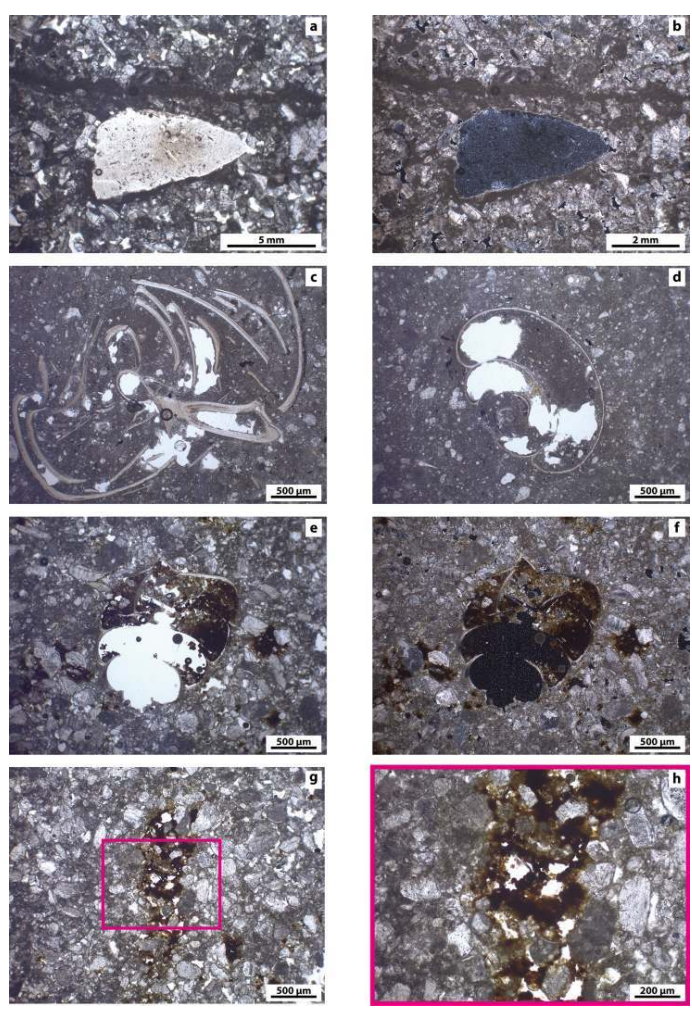

Villa 2017, p. 201.

\section{Fouilles archéologiques}

Le niveau SLM-37 avait été fouillé sur quelques mètres carrés lors des fouilles Radmilli dans les années 1950 et avait livré 502 pièces, dont cinq bifaces, d'après les décomptes disponibles. C'est ce niveau qui a fait la célébrité de Valle Giumentina : c'est en raison de la présence de bifaces que A.M. Radmili a attribué le site à l'Acheuléen (la culture du Paléolithique inférieur à bifaces) et par extension, les autres niveaux d'occupation, sans bifaces mais à industries à éclats, ont été attribués à la culture du Clactonien. 

surface restreinte de $7 \mathrm{~m}^{2}$. Il avait pu être atteint directement moyennant quelques aménagements de sécurité, suite à un glissement de terrain qui avait emporté la partie supérieure du terrain en cet endroit. Il convenait toutefois d'atteindre la base du niveau sur l'intégralité de l'aire de fouille. En effet, alors que nous pensions ce niveau SLM-37 extrêmement compact et épais de quelques centimètres seulement, nous avons découvert en fin de campagne 2016 des vestiges sporadiques situés à $10 \mathrm{~cm}$ plus en profondeur que le reste des pièces. Faute de temps, nous n'avions pu fouiller que $0,5 \mathrm{~m}^{2}$ jusqu'aux sables blancs du niveau sous-jacent SFL. Une pièce de grandes dimensions était apparue. La couche SLM-37 s'avérait ainsi épaisse de plus de $50 \mathrm{~cm}$. En 2017, cette fouille restreinte du niveau SLM-37 en VV3 a donc été poursuivie jusqu'à la base de l'horizon brun sur le reste de la plateforme de fouille. Neuf éclats seulement sont apparus, extrêmement dispersés, dont il conviendra d'expliquer la présence sporadique. Les informations récoltées sur la coupe VV3 depuis 2015 nous avaient donné beaucoup d'indices pour mettre en œuvre la fouille de ce niveau dans l'aire principale VV1.

Sur la coupe principale VV1 où une plateforme plus ample a été aménagée depuis 2013, les niveaux supérieurs ALB-42, LDP-41 et LABM-40 ont été fouillés les années précédentes. L'objectif pour 2017 était donc d'ouvrir la fouille du niveau SLM-37, sur une surface plus conséquente que celle de la coupe VV3. Les mesures de sécurité nous ont permis ici de mettre au jour $35 \mathrm{~m}^{2}$ de ce niveau, épais d'une vingtaine de centimètres (fig. 4).

Fig. 4 - Valle Giumentina 2017 niveau SLM-37 en VV1 : a. plan des vestiges ; b. position de l'aire de fouille sur le modèle numérique de terrain ; $c$. vue de la fouille en cours.

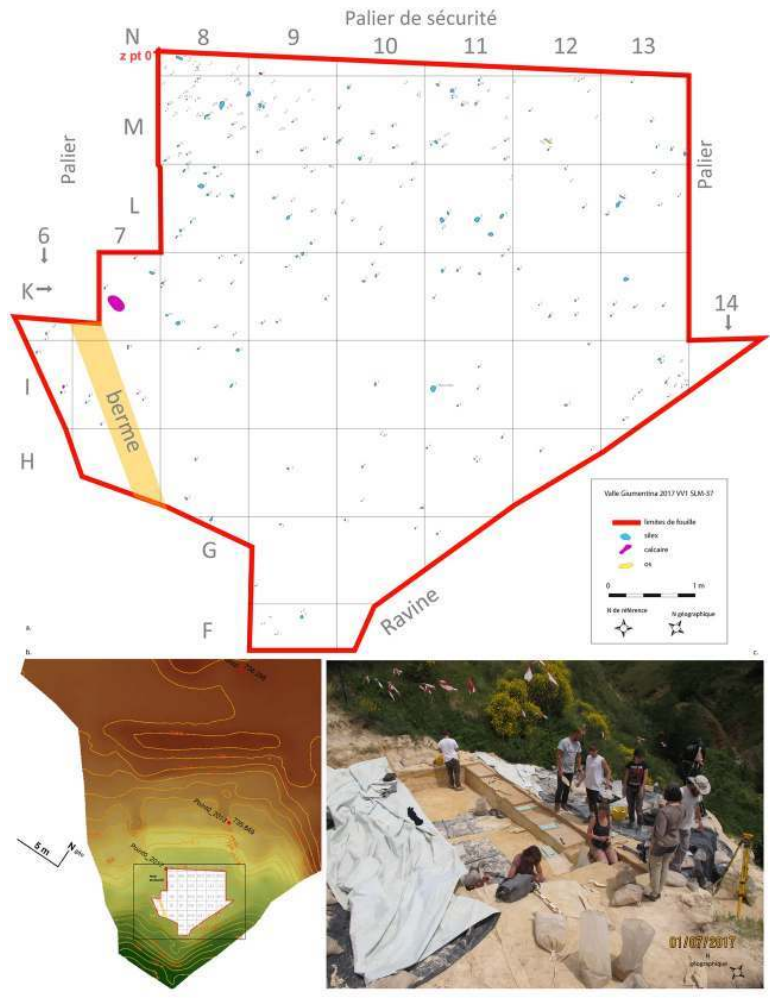

a. E. Nicoud ; b. G. Davtian ; c. EFR/E. Nicoud.

Les études sur l'industrie et la faune se poursuivent en laboratoire : aussi cet exposé est-il extrêmement liminaire. La fouille de la plateforme VV1 de $35 \mathrm{~m}^{2}$ a livré 250 artefacts 
lithiques (hors fugaces), essentiellement en silex et plusieurs petits galets calcaires sous forme d'éclats gélifs. Trois restes osseux sont présents extrêmement abîmés (fig. 5). Le plus remarquable est un fragment de côte de Cervus elaphus (identification par M.A. Rossi et $\mathrm{P}$. Mazza). Les sédiments résiduels ont été entièrement tamisés par quart de $\mathrm{m}^{2}$, livrant 439 unités de refus de tamis dont 15 contenant des restes osseux très fragmentaires.

Avant la découverte d'indices de productions bifaciales dans le niveau supérieur LABM-40, le niveau 37 se distinguait du reste de la séquence archéologique, puisqu'il témoigne d'une production bifaciale. Les éclats présents sont ainsi pour la plupart très fins. Si certains portent les caractéristiques d'éclats de façonnage bifacial (profil courbe, talon arraché), d'autres pourraient aussi provenir d'un débitage : les talons sont épais, inclinés et lisses. Les négatifs antérieurs sont uni- ou bipolaires. Dans l'industrie du niveau 37 découverte par A.M. Radmilli, un éclat épais semble avoir été utilisé en nucléus : sa face inférieure sert de plan de frappe pour un éclat qui crée une encoche sur le support et un petit éclat est produit aux dépens de la surface inférieure. Deux grands éclats-supports portent quelques enlèvements de retouche marginale ${ }^{12}$.

Fig. 5 - Valle Giumentina 2017 SLM-37 : exemples de vestiges découverts en VV1, certains sont en position verticale.

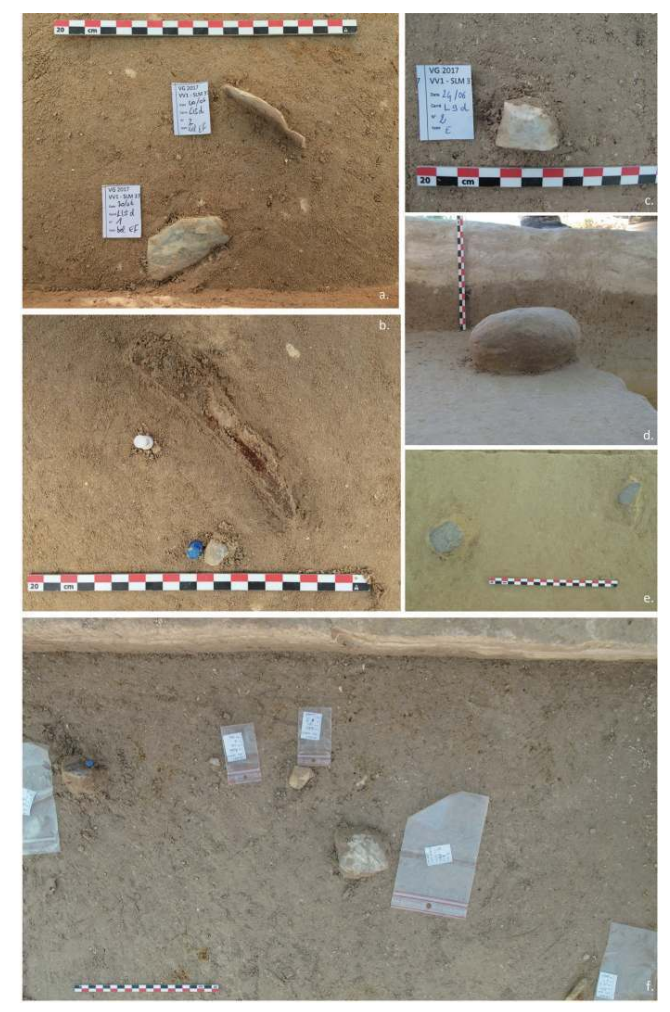

EFR/E. Nicoud.

Un examen macroscopique préliminaire des pièces du niveau SLM-37 a été réalisé par J. Guibert-Cardin, en particulier de la pièce bifaciale I11-3 (fig. 6) qui livre des informations encourageantes en vue d'une étude tracéologique. En effet, le biface présente un aspect frais avec des altérations taphonomiques peu développées. À la loupe binoculaire, il apparaît un lustré de sol peu développé, faiblement réfléchissant et présent sur toute la surface. Une patine blanche se développe à l'air libre, après le lavage de la pièce. Quelques encroûtements sont présents dans les parties concaves de la surface mais la plupart a 
disparu après 20 minutes dans la cuve à ultra-sons. Les encroûtements restants sont principalement situés dans les écrasements de la base. Par ailleurs, les bright spots sont très rares et un faible émoussé arrondi est présent sur les arêtes et les bords. Au microscope, le léger lustré de sol à la surface de la pièce est confirmé, et il est un peu plus développé dans les zones où se trouvent les encroûtements. Sur les arêtes, un faible émoussé est associé à un poli plat taphonomique peu développé, discontinu, à coalescence dure et aux limites nettes. Un premier moulage des bords a été réalisé juste après le lavage de la pièce, avant que la patine ne se développe trop puis un second moulage a été effectué après avoir passé la pièce à la cuve à ultra-sons et retiré la plupart des encroûtements. La comparaison de la surface sur les moulages avant et après la formation de la patine ne montre pas de différences (observations jusqu'à × 200).

Fig. 6 - Valle Giumentina 2016, niveau SLM-37 en VV1. Pièce bifaciale I1 1-3 découverte en 2017 semblable en dimensions et facture à la pièce bifaciale étudiée et provenant des fouilles Radmilli (cf. Nicoud et al. 2015, p. 18).

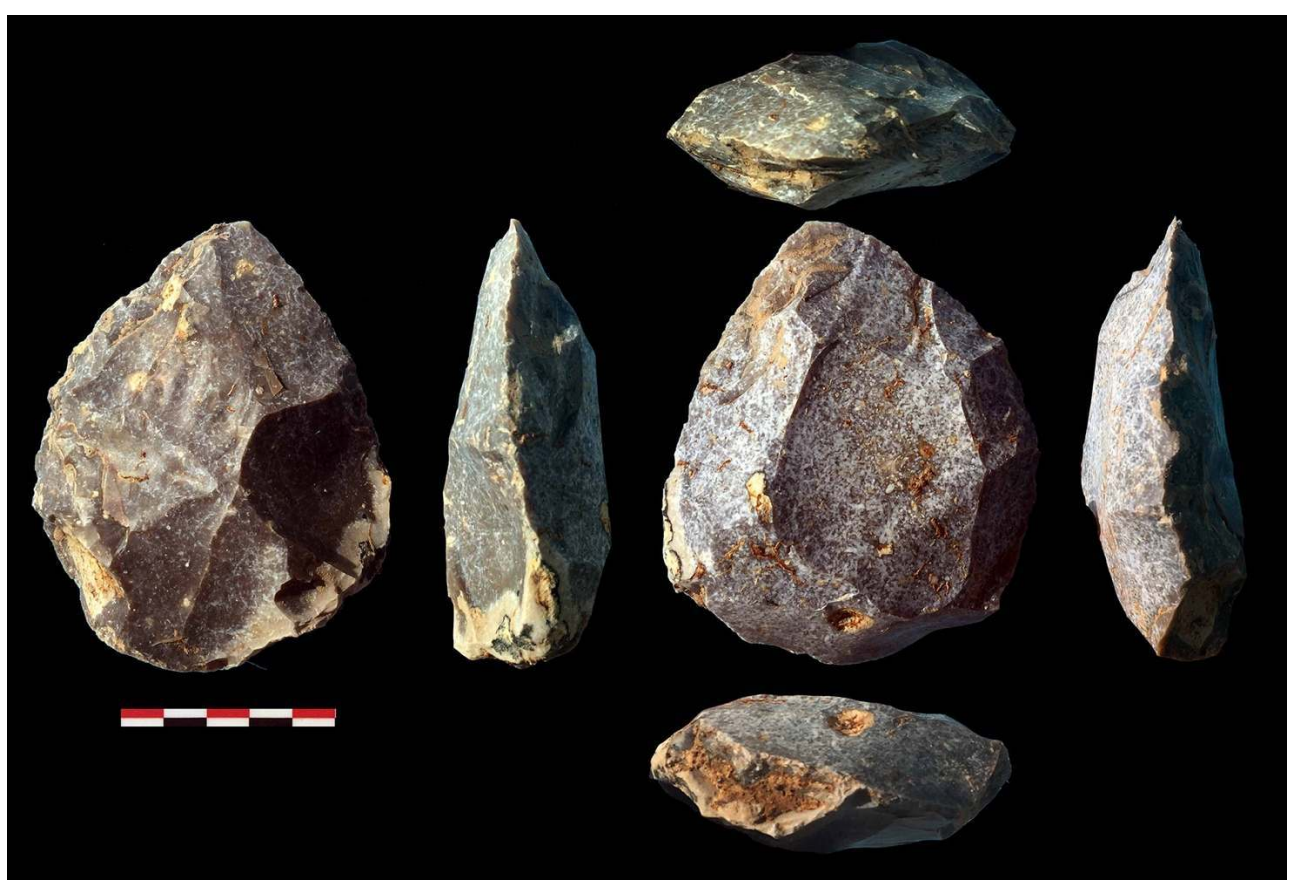

EFR/E. Nicoud.

\section{Perspectives}

20 Avec l'aboutissement des travaux doctoraux sur le contexte géologique, paléoenvironnemental et les datations de Valle Giumentina ${ }^{13}$, un nouveau cadre géochronologique est disponible, et d'ores et déjà publié sous diverses formes ${ }^{14}$. Il permet de poursuivre désormais l'étude technologique des industries sereinement et de replacer ces faits techniques dans un contexte assuré. Une nouvelle thèse de doctorat débute, menée par Juliette Guibert-Cardin à l'université Côte d'Azur sur l'étude tracéologique des industries de Valle Giumentina. L'étude archéologique est désormais notre priorité, pour discourir des techniques employées par les groupes humains à Valle Giumentina, leur évolution possible et leur place dans le contexte européen et méditerranéen du Paléolithique ancien. Nous nous attachons également à décrire les réponses anthropiques 
face aux variations climatiques mises en évidence par l'étude paléoenvironnementale. Pour cela nous devons établir quelles ressources minérales, animales ou végétales étaient à disposition tant en période glaciaire qu'interglaciaire pour reconnaître plus avant les modalités de subsistance des groupes, leurs modalités d'occupation du territoire. L'analyse des matières premières utilisées sur le site se poursuit (A. Tomasso).

En 2018, sur le terrain, l'objectif est d'abaisser les plateformes de fouille pour atteindre en VV1 le niveau découvert lors du nettoyage de la coupe en 2013 nommé «LAC 34 » et en VV3, le niveau SFL-36 sous-jacent au niveau SLM-37. Des relevés topographiques et photogrammétriques seront poursuivis l'an prochain, de façon à produire une cartographie de qualité et pouvoir restituer nos informations par des images en 3D. Les publications et autres présentations des résultats se poursuivent.

\section{BIBLIOGRAPHIE}

Degeai et al. 2018 = J.-Ph. Degeai, V. Villa, C. Chaussé, A. Pereira, S. Nomade, D. Aureli, M. Pagli, É. Nicoud, Chemical weathering of palaeosols from the Lower Palaeolithic site of Valle Giumentina, central Italy, dans Quaternary Science Reviews, 183, 2018, p. 88-109.

Demangeot - Radmilli 1966 = J. Demangeot, A.M. Radmilli, Le gisement paléolithique de Valle Giumentina (Apennin central) et ses problèmes, dans Eiszeitalter und Gegenwart, 17, 1966, p. 159-299.

Limondin-Lozouet et al., 2017 = N. Limondin-Lozouet, V. Villa, A. Pereira, S. Nomade, J.-J. Bahain, E. Stoetzel, D. Aureli, M. Pagli, E. Nicoud, Middle Pleistocene Molluscan Fauna from Central Italy at Valle Giumentina (Abruzzo): palaeoenvironmental, biostratigraphical and biogeographical implications dans Quaternary Science Reviews, 156, 2017, p. 135-149.

Nicoud et al. 2015 = É. Nicoud, D. Aureli, M. Pagli, Comportements techniques au Pléistocène moyen en Italie : nouvelles recherches sur l'industrie lithique et le site de Valle Giumentina (Abruzzes), dans MEFRA, 127-1, 2015 [en ligne]. URL : http://mefra.revues.org/2659

Nicoud et al. 2016 = É. Nicoud, D. Aureli, M. Pagli, V. Villa, C. Chaussé, S. Agostini, J.-J. Bahain, G. Boschian, J.-P. Degeai, F. Fusco, B. Giaccio, M. Hernandez, C. Kuzucuoglu, C. Lahaye, C. Lemorini, N. Limondin-Lozouet, P. Mazza, N. Mercier, S. Nomade, A. Pereira, V. Robert, M.A. Rossi, C. Virmoux, A. Zupancich, Preliminary data from Valle Giumentina Pleistocene site (Abruzzo, Central Italy): a new approach to a Clactonian and Acheulian sequence, dans Quaternary International, 409, 2016, p. 182-194.

Nicoud et al. 2017 = É. Nicoud, D. Aureli, M. Pagli, Valle Giumentina (Abruzzes, Italie), dans Chronique des activités archéologiques de l'École française de Rome [en ligne]. URL : http:// journals.openedition.org/cefr/1656

Pereira 2017 = A. Pereira, Apport de la datation ${ }^{40} \mathrm{Ar} /{ }^{39} \mathrm{Ar}$ à la compréhension de l'évolution culturelle des pré-néanderthaliens en Italie centrale et méridionale entre 750 et $250 \mathrm{ka}$, thèse de doctorat, 2017, Muséum national d'histoire naturelle, Università di Ferrara, École française de Rome. 
Villa 2017 = V. Villa, Environnements et occupations paléolithiques d'Italie centrale : la longue séquence pléistocène moyen de Valle Giumentina, thèse de doctorat, 2017, université Paris 1 PanthéonSorbonne, Università di Pisa, École française de Rome.

Villa et al. 2016 = V. Villa, A. Pereira, C. Chaussé, S. Nomade, F. Fusco, N. Limondin-Lozouet, J.-P. Degeai, V. Robert, C. Kuzucuoglu, G. Boschian, D. Aureli, M. Pagli, É. Nicoud, A MIS 15-MIS 12 record of environmental changes and Lower Palaeolithic occupation from Valle Giumentina, central Italy, dans Quaternary Science Reviews, 151, 2016, p. 160-184.

\section{NOTES}

1. Demangeot - Radmilli 1966.

2. Nicoud et al. 2016 et 2017.

3. Villa et al. 2016 ; Degeai et al. 2018.

4. Villa 2017 ; Pereira 2017.

5. Limondin-Lozouet et al. 2017.

6. Villa 2017.

7. La description des différents niveaux est disponible dans Villa et al. 2016 et dans les Chroniques précédentes.

8. Limondin-Lozouet et al. 2017.

9. Limondin-Lozouet et al. 2017.

10. Villa et al. 2016 ; Pereira 2017 ; Villa 2017.

11. Limondin-Lozouet et al. 2017 ; Villa 2017.

12. Nicoud et al. 2015.

13. Villa 2017 ; Pereira 2017.

14. Par ex. Villa et al. 2016 ; Degeai et al. 2018.

\section{INDEX}

Mots-clés : Paléolithique, Abruzzes, Pléistocène moyen, Valle Giumentina, technologie lithique institutions Institutions : École française de Rome, Soprintendenza per i beni archeologici dell'Abruzzo, INRAP, CNRS (CEPAM UMR 7264, ArScAn-AnTeT UMR 7041, LGP UMR 8591, ASM UMR 5140, IRAMAT UMR 5060, LSCE UMR 8212 et HNHP UMR 7194), université Bordeaux Montaigne, université Paris 1 Panthéon-Sorbonne (ED de géographie de Paris), Commissariat à l'énergie atomique, Università degli studi di Bologna Ravenna, di Siena (preistoria e antropologia), di Pisa (biologia), di Firenze (scienze della Terra), di Roma La Sapienza, CNR, CGT Group Siena, Parco nazionale della Majella, Fondation Pescarabruzzo, Comune di Abbateggio, Comune di Caramanico Terme, Museo delle Genti d'Abruzzo, Archeoclub di Pescara 


\section{AUTEURS}

\section{ÉLISA NICOUD}

CNRS - UMR 7264 CEPAM, université Côte d'Azur - elisa.nicoud[at]cepam.cnrs.fr

\section{VALENTINA VILLA}

Post-doctorante, Laboratoire UMR 8591 LGP Meudon - valentina.villa@lgp.cnrs.fr

\section{GOURGUÈNE DAVTIAN}

CNRS - UMR 7264 CEPAM, université Côte d'Azur - gourguen.davtian@cepam.cnrs.fr JULIETTE GUIBERT-CARDIN

Doctorante, université Côte d'Azur, UMR 7264 CEPAM - juliette.guibert-cardin@cepam.cnrs.fr 